\title{
Ammann Bars and Quasicrystals
}

\author{
Thomas Stehling
}

Institut für Mathematik, Universität Dortmund, Postfach 500500 , 4600 Dortmund, Federal Republic of Germany

\begin{abstract}
In 1988 Danzer [3] constructed a family of four tetrahedra which allows-with certain matching conditions-only aperiodic tilings. By analogy with the Ammann bars of planar Penrose tilings we define Ammann bars in space in the form of planar Penrose tilings we define Ammann bars in space in the form of plane sections of the four tetrahedra. If we require that the plane sections continue as planes across the faces of the tilings, we obtain an alternative matching condition, thus answering a question of Danzer.
\end{abstract}

\section{Introduction}

The discovery of electron diffraction patterns of an Al-Mn alloy with icosahedral symmetry by Shechtman et al. [10] in 1984 has initiated not only much activity in experimental and theoretical physics, but has also had a stimulating effect in several areas of mathematics. The alloys exhibit long-range orientational order but no translational symmetry. These novel kinds of structures, intermediate between crystalline and amorphous, have been termed quasicrystals [7]. Meanwhile, several other quasicrystals have been described, some whith different rotational symmetry (see e.g., [1] and [4]) and many with different chemical composition.

On the theoretical side, several methods for constructing such structures have been developed (for a survey see, e.g., [9]). One of these methods is the principle of inflation/deflation, which is described in [5] in the case of the famous planar Penrose tilings [8].

In 1988 Danzer [3] succeeded in finding a family $\mathbb{F}_{1}:=\{A, B, C, K\}$ of four tetrahedra, derived from the regular icosahedron, which admits-with certain matching conditions - the principle of inflation/deflation. Homothets in the ratio $2: 1$ of the four tetrahedra are described in Table 1. For each pair of vertices, the dihedral angle at the corresponding edge and the edgelength are specified. 
Table 1

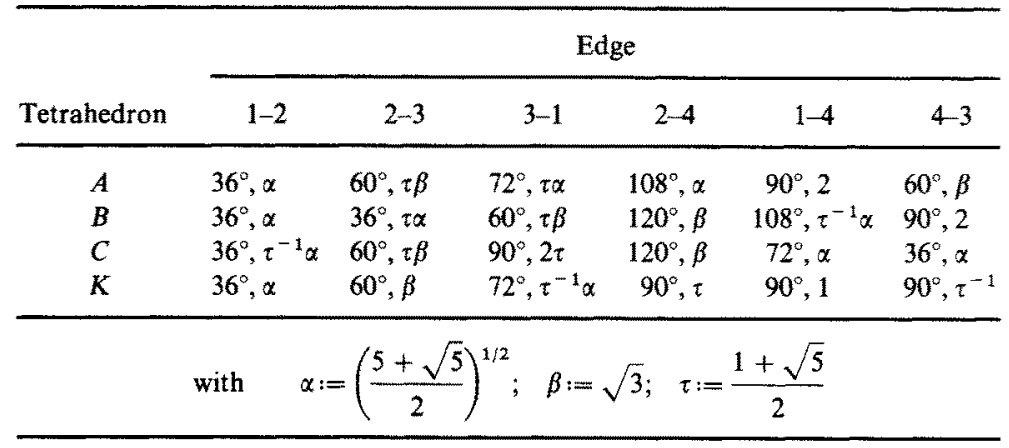

Let us describe Danzer's matching condition with the help of Fig. 3(a), which shows the facets $A_{1}, \ldots, A_{4}, B_{1}, \ldots, B_{4}, C_{1}, \ldots, C_{4}, K_{1}, \ldots, K_{4}$ of $A, B, C$, and $K$. The edges of the first seven facets in Fig. 3(a) all have dihedral angles other than $90^{\circ}$. Facets $A_{4}$ and $B_{4}$ (resp. $C_{1}$ and $A_{1}$ ) (resp. $K_{4}, C_{3}$, and $B_{4}$ ) are congruent. In each of the other nine facets there exists at least one edge with a $90^{\circ}$ angle, for instance, the edge 1-4 in $A_{2}, \ldots$ (see Table 1).

Danzer first demands that the tilings have to be face-to-face. Therefore, we only can juxtapose the tetrahedra in congruent facets. Furthermore, Danzer demands that if $\Delta$ is a facet of a tetrahedron $T$ with at last one $90^{\circ}$ edge, then the mirror image of $T$ has to be on the other side of $\Delta$. Therefore, it is forbidden to juxtapose $A$ and $B$ in the facets $A_{2}$ (resp. $B_{1}$ ), even if the facets are congruent.

In connection with the matching condition Danzer asked as an open problem whether there exist three-dimensional analogues of the Ammann bars of planar Penrose tilings. Our aim in this paper is to introduce Ammann bars in Danzer tilings for which the resulting matching condition is equivalent to that given by Danzer.

\section{Ammann Bars in Planar Penrose Tilings}

Let us first give a brief introduction to Ammann bars in the case of the not so disseminated trinalge version of planar Penrose tilings. For a more detailed description (for example, of the famous dart and kite, the Penrose rhombs, the triangle version, ...) we refer to the book by Grünbaum and Shephard [5].

The tiles given in Fig. 1 are triangles which allow periodic tilings. If we demand that colors of vertices as well as lengths and orientations of edges must match, we get aperiodic tilings which can be generated by the principle of inflation/deflation (see [5]).

Another way of marking the tiles is by means of straight line segments which must continue as straight lines across the edges of the tiling. In Fig. 1 we show how the line segments may be defined for the triangles, yielding equivalent matching conditions. Figure 2 shows a tiling by Penrose triangles with the tiles marked by lines as in Fig. 1. These lines are known as Ammann bars after their 


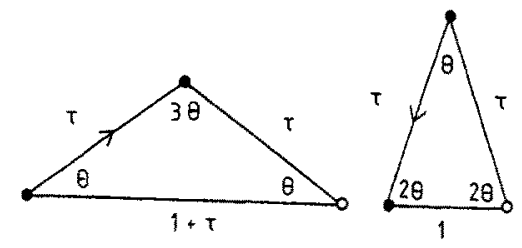

$$
\theta=\frac{\pi}{5}
$$
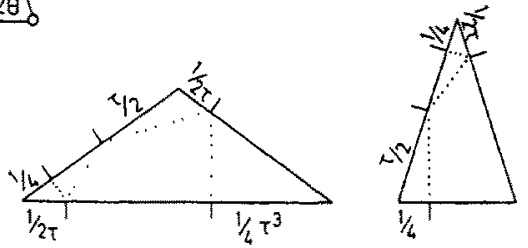

Fig. 1

originator, and they are of great importance, for instance in the theory of musical sequences, wholes, empires, or forced tiles in aperiodic tilings (see Section 10.6 of [5]).

\section{Ammann Bars in Three-Dimensional Danzer Tilings}

The Ammann bars in two-dimensional tilings are lines. Our idea was to introduce an anologue for the three-dimensional tilings which consists of planes. Therefore we have searched for certain plane sections within the four prototiles of Danzer which must continue as planes across the facets of the tiling.

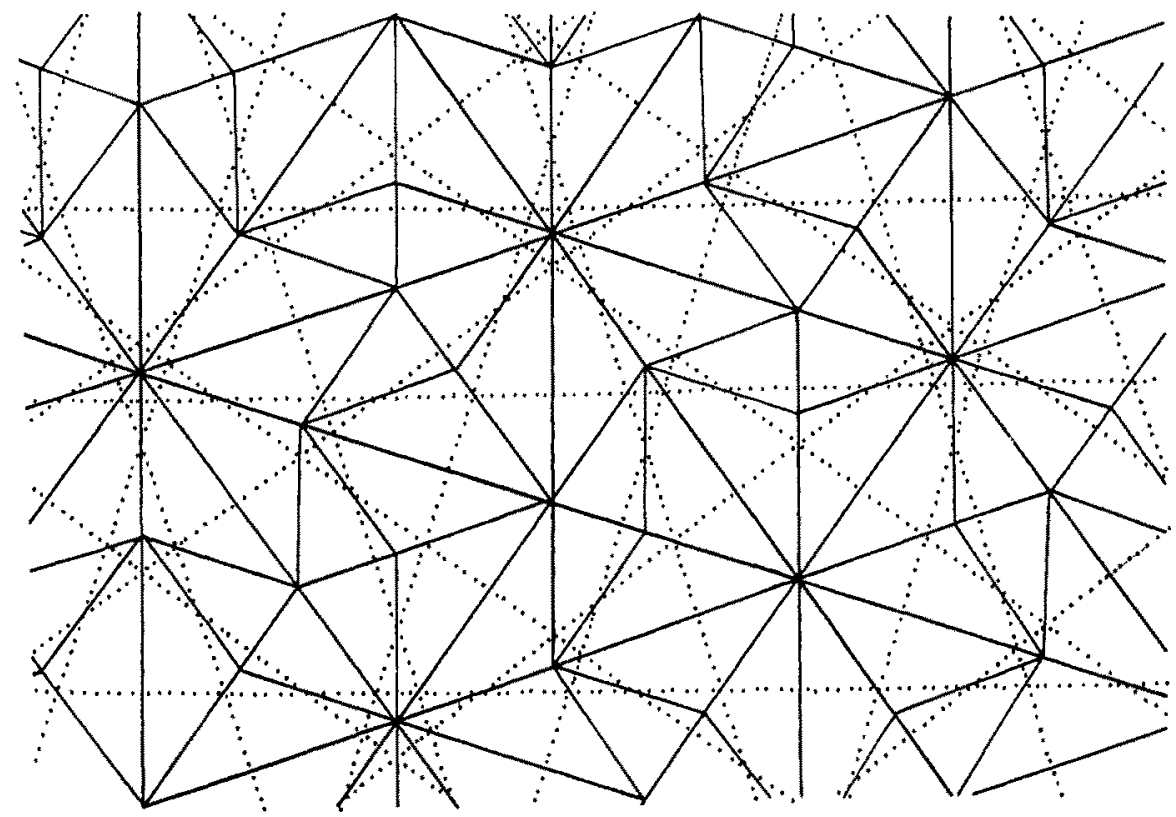

Fig. 2 

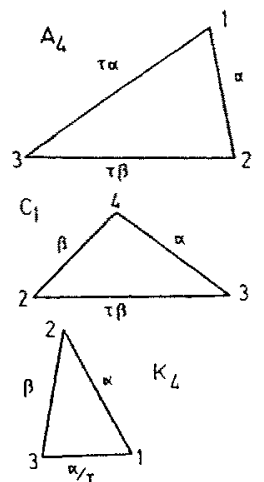

(i) The seven facets without $90^{\circ}$ edge
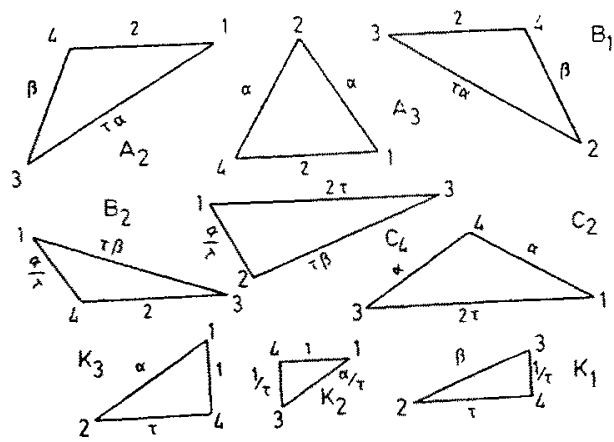

(ii) The nine facets with $90^{\circ}$ edge

(a)
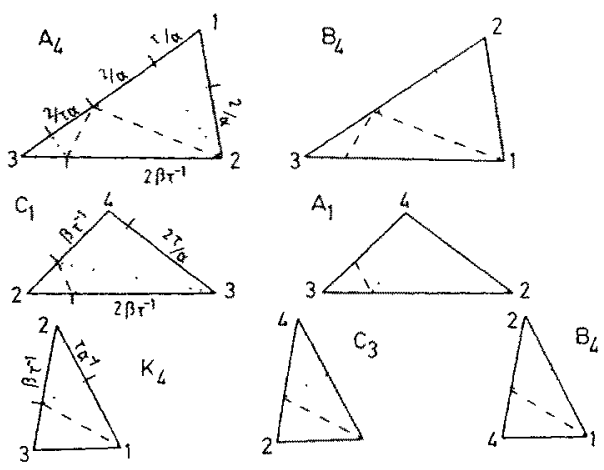

(i) The seven facets without $90^{\circ}$ edge
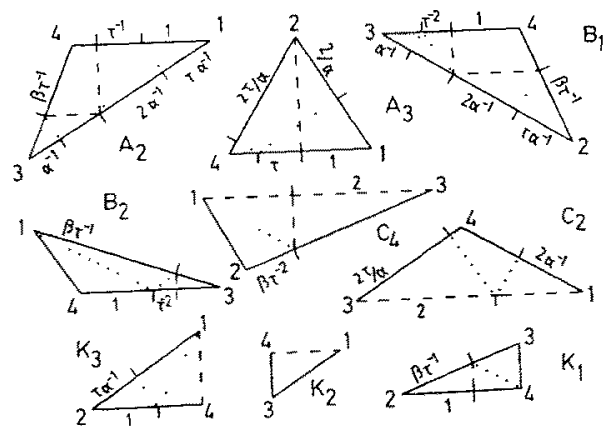

(ii) The nine' facets with $90^{\circ}$ edge

(b)

Fig. 3

The dotted or dashed line segments in Fig. 3(b) indicate the intersection of the planes (Ammann bars) with the facets of $A, B, C$, and $K$. The seven plane sections $a b c, i h 2, a b d, b d e f, d e g, a d 2, d i 2$ of $A$, the seven plane sections $b c 1, f g h$, de1, def, $d f g, a c d, c d 1$ of $B$, the four plane sections $c d 1, b c d e, a b c, d 13$ of $C$, and the three plane sections $a b c, b c 1, c 14$, of $K$ are shown in Fig. 4. For a metric description we refer to Table 2 .

Let $\Delta$ be any facet of a tetrahedron $T \in\{A, B, C, K\}$. In order to prove the equivalence of the matching condition defined above in terms of Ammann bars and the matching condition given by Danzer, we have to show that the Ammann bars continue if we juxtapose $T$ and the mirror image of $T$ in $\Delta$. If $\Delta$ has a $90^{\circ}$ edge (Fig. 3(b)(ii)) we have to show that this is the only way of juxtapositioning with continuing Ammann bars. Furthermore, we have to show that two different tetrahedra of Danzer can be juxtaposed with continuing Ammann bars in congruent facets if and only if the facet has no $90^{\circ}$ edge (see Fig. 3(b)(i)). For example, if we juxtapose $A$ and $B$ in $A_{4}$ (resp. $B_{4}$ ) the planar sections of $A$ and $B$ have to continue. 

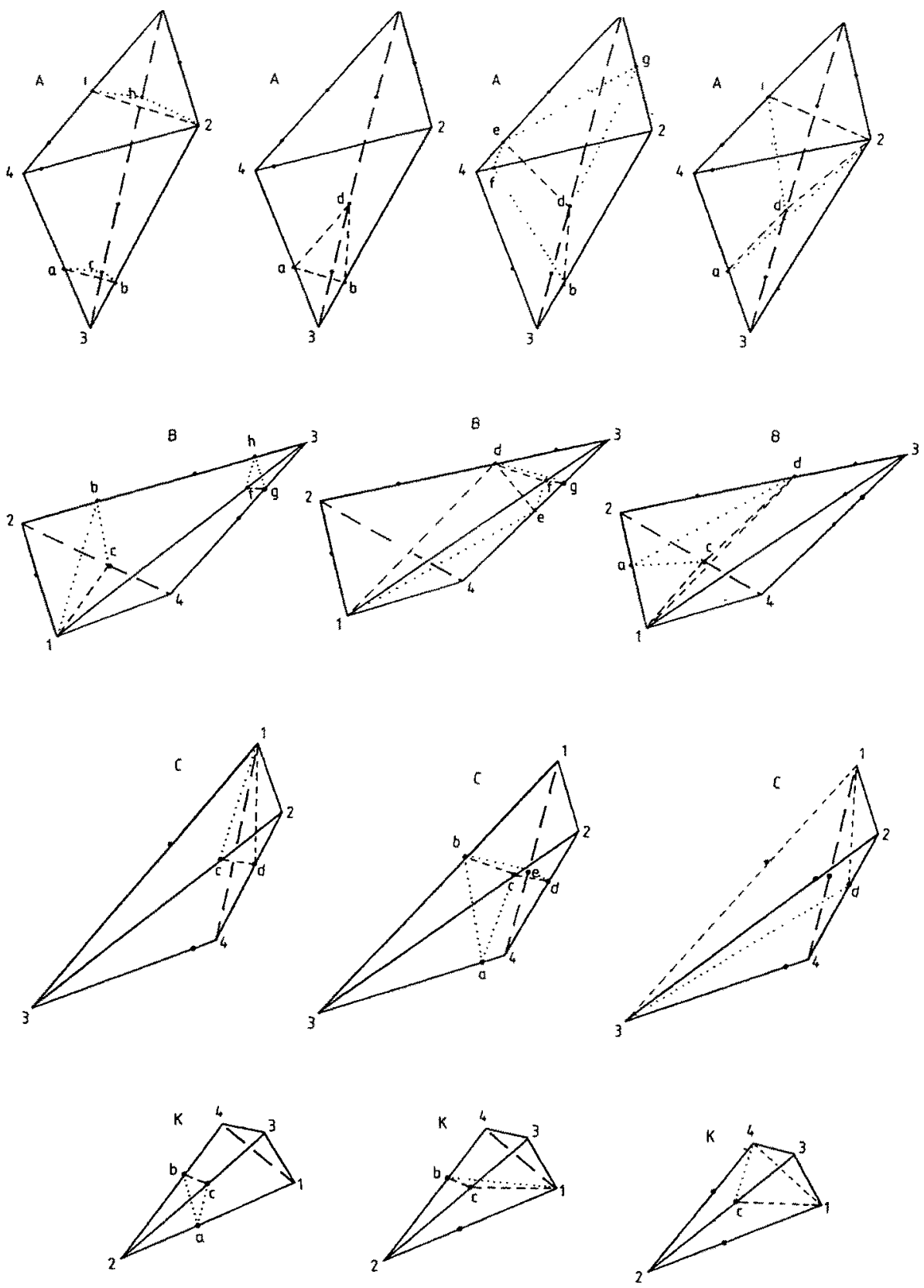

Fig. 4 
Table 2

\section{Tetrahedra A}

\section{Coordinates:}

1: $\left(0, \tau, \tau^{2}\right)$

a: $\left(-\tau^{-3}, 0, \tau^{-1}\right)$

2: $\left(1,0, \tau^{2}\right)$

3: $(0,0,0)$

c: $\alpha^{-1}(0,1, \tau)$

$4:\left(-\tau^{-1}, 0, \tau\right)$

e: $\frac{1}{2}\left(-1, \tau^{-1}, 2+\tau\right)$

f: $\alpha^{-2}\left(\tau, 0,2+\tau^{3}\right)$

$\mathrm{g}: \alpha^{-2}\left(\tau, 2 \tau, \tau^{2} \alpha^{2}\right)$

d: $\left(0, \tau^{-1}, 1\right)$

i: $\frac{1}{2}\left(-\tau^{-1}, \tau, \alpha^{2}\right)$

Normal vectors to facets:

$A_{1}:(0,2,0) \quad A_{2}:\left(-\tau, 1,-\tau^{-1}\right) \quad A_{3}:\left(-1,-\tau^{-1}, \tau\right) \quad A_{4}:\left(\tau, 1, \tau^{-1}\right)$

Normal vectors to plane sections:

$a b c:(0,1, \tau) \quad$ abd: $(0,1,-\tau) \quad$ bdef: $(\tau, 0,1) \quad$ ad2: $(\tau, 0,-1)$

deg: $(1,-\tau, 0) \quad$ di2: $(1, \tau, 0) \quad$ ih $2:(0,1, \tau)$

Dihedral angles between facets and plane sections:

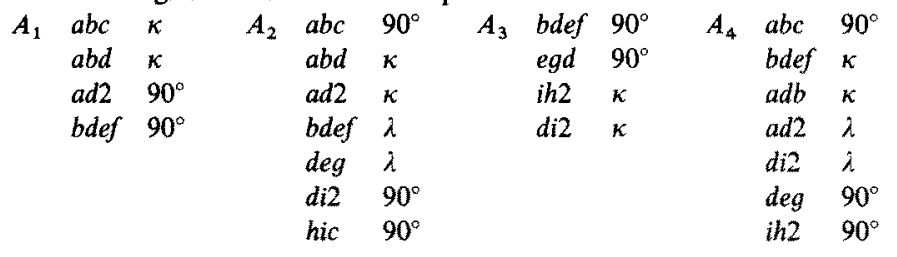

Tetrahedron B

Coordinates:

1: $(0,0,0)$

a: $\alpha^{-2}(-2,2 \tau, 0)$

$2:(-1, \tau, 0)$

3: $(\tau, \tau, \tau)$

c: $\left(0,2 \tau^{-1}, 0\right)$

h: $\alpha^{-2}\left(0, \tau^{3}, \tau^{4}\right)$

e: $\frac{1}{2}\left(2 \tau-1, \tau^{2}, \tau\right)$

b: $\alpha^{-2}\left(-1, \tau \alpha^{2}, \tau\right)$

g: $\frac{1}{2}\left(\tau^{2}, 3 \tau-2,2 \tau-1\right)$

4: $\left(\tau^{-1}, 1,0\right)$

f: $2 \tau^{-1}(1,1,1)$

$\mathrm{d}:\left(\tau^{-1}, \tau, 1\right)$

Normal vectors to facets:

$B_{1}:\left(\tau^{-1}, \tau,-1\right) \quad B_{2}:\left(\tau,-1,-\tau^{-1}\right) \quad B_{3}:(0,0,2) \quad B_{4}:\left(1, \tau^{-1},-\tau\right)$

Normal vectors to plane sections:

acd: $(-1, \tau, 0) \quad b c 1:(\tau, 0,1) \quad c d 1:(-\tau, 0,1) \quad$ de1: $(0,-1, \tau)$

$\operatorname{def}:(1, \tau, 0) \quad d f g:(0,1, \tau) \quad f g h:(\tau, 0,1)$

Dihedral angles between facets and plane sections:

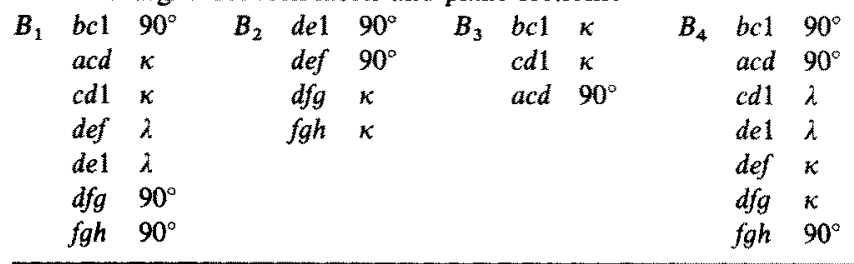

Tetrahedron $\mathrm{C}$

Coordinates:
$1:\left(1, \tau^{2}, \tau\right)$
a: $\alpha^{-2}\left(2 \tau, 2 \tau^{2}, 0\right)$
e: $\alpha^{-1}\left(\alpha^{2}, 4 \tau+1, \tau^{2}\right)$
2: $(\tau, \tau, \tau)$
3: $(0,0,0)$
b: $\left(\tau^{-1}, \tau, 1\right)$
c: $2 \tau^{-1}(1,1,1)$
4: $(1, \tau, 0)$
d: $\left(2-\tau^{-1}, \tau, 1\right)$

Normal vectors to facets:
$C_{1}:\left(\tau,-1,-\tau^{-1}\right)$
$C_{2}:\left(\tau,-1, \tau^{-1}\right)$
$C_{3}:\left(\tau, 1,-\tau^{-1}\right)$
$C_{4}:\left(1, \tau^{-1},-\tau\right)$

Normal vectors to plane sections:

$a b c:(1, \tau, 0) \quad b c d e:(0,1, \tau) \quad c d 1:(\tau, 0,1) \quad d 13:(0,-1, \tau)$

Dihedral angles between facets and plane sections:

$\begin{array}{llllllllllll}C_{1} & b c d e & \kappa & C_{2} & d 13 & \kappa & C_{3} & b c d e & 90^{\circ} & C_{4} & d 13 & \lambda \\ & c d 1 & \kappa & & a b c & 90^{\circ} & & c d 1 & \kappa & & c d 1 & 90^{\circ} \\ d 13 & 90^{\circ} & & b c d e & 90^{\circ} & & d 13 & \kappa & & b c d e & \kappa \\ a b c & 90^{\circ} & & & & & & & & a b c & \kappa\end{array}$


Table 2 (continued)

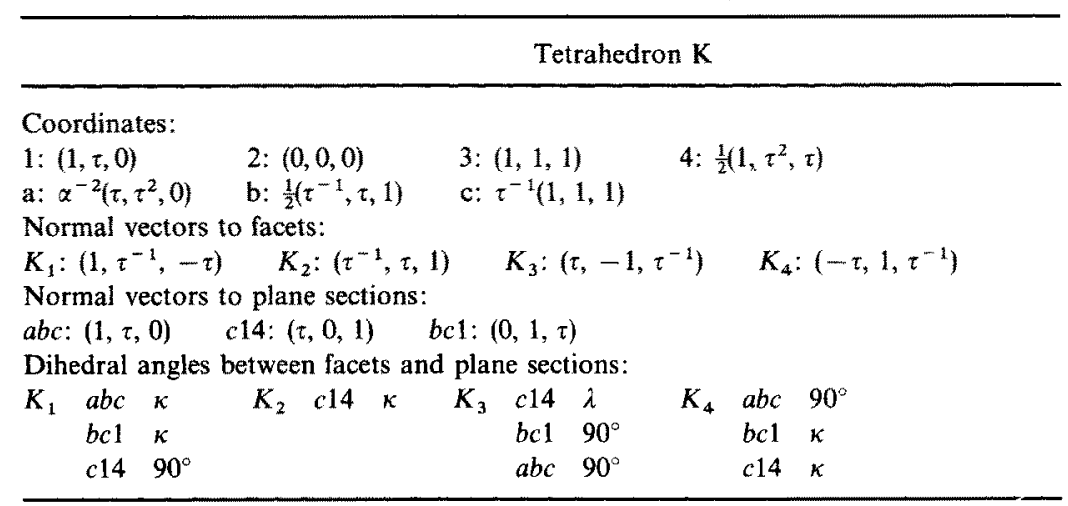

Theorem. The matching conditions defined in terms of the Ammann bars above are equivalent to the matching conditions given by Danzer.

Proof. In order to prove the equivalence of the matching conditions we first choose suitable coordinates for the four tetrahedra $A, B, C$, and $K$ and their inscribed Ammann bars. Next we compute the dihedral angles between the Ammann bars and the facets they intersect. These angles are listed in Table 2, where we define

$$
\kappa:=\arccos \left(\alpha^{-1}\right) \text { and } \lambda:=\arccos \left(\tau \alpha^{-1}\right)=90^{\circ}-\kappa .
$$

Figure 4 shows that each dotted line segment lies on exactly one Ammann bar. Since the plane sections must continue as planes across the facets of the tiling, the dihedral angle between the facets and the plane sections corresponding to the dotted line segments must be $90^{\circ}$, in agreement with Table 2 (see Fig. 5). Let us now consider the dashed intersections. Here the facets intersect with two different Ammann bars (compare Figs. 4 and 5). Table 2 shows that the dihedral angles $\xi_{1}$ and $\xi_{2}$ are the same for each dashed line.

In each of the nine facets without $90^{\circ}$ edges shown in Fig. 3(b) the Ammann bars induce identical structures with the same dihedral angles (see Table 2) for congruent facets. In the case of the nine facets having $90^{\circ}$ edges, the Ammann bars induce different structures, particularly in the case of $A_{2}$ and $B_{1}$, which are
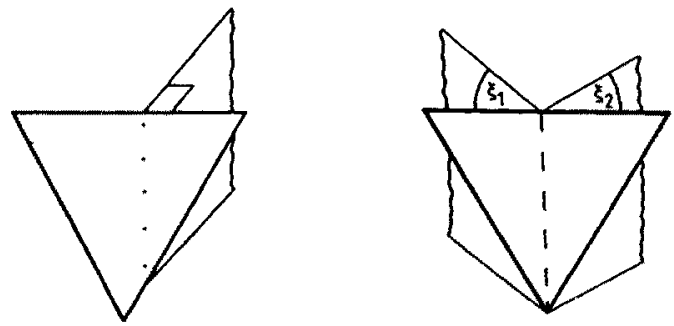

Fig. 5 


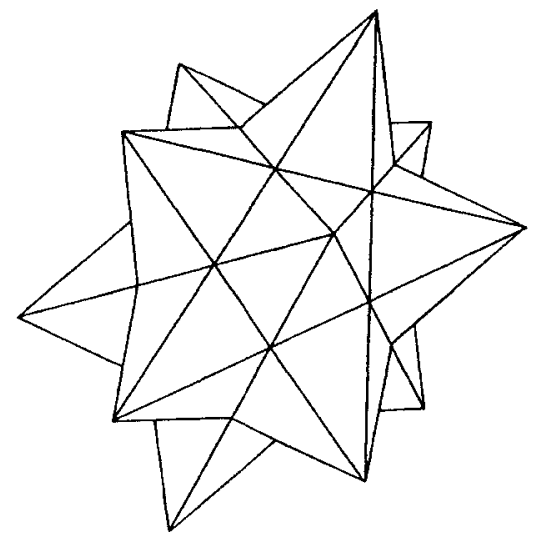

Fig. 6

congruent but not equivalent. In $C_{2}$ and $C_{4}$ (resp. in $K_{2}$ and $K_{3}$ ) the intersection of two Ammann bars with the facet is a $90^{\circ}$ edge. Because of the definition of Danzer's matching condition there must be exactly four tetrahedra of type $C$ (resp. $K$ ), which come together along the $90^{\circ}$ edge. Table 2 shows that the plane sections do continue in these cases. Thus the theorem is proved.

\section{Remarks}

The Ammann bars in Fig. 2, considered separately, induce a tiling of the plane. Similarly, there arises the problem of determining the prototiles of the threedimensional tiling which is induced by the Ammann bars in space. For example, the three-dimensional analogue of the pentagram is a starpolyhedron which occurs if the $120 \mathrm{~K}$ 's have one vertex in common (Fig. 6).

The existence of Ammann bars in three-dimensional tilings may prove useful in further investigations. For instance, the theory of musical sequences, wholes, empires, forced tiles, etc. (see [5]), should be transferrable to the spatial case.

\section{References}

1. L. Bendersky, Quasicrystals with one-dimensional translational symmetry and a tenfold rotational axis, Phys. Rev. Lett. 55 (1985), 1461-1463.

2. N. G. de Bruijn, Algebraic theory of Penrose's non-periodic tilings, Nederl. Akad. Wetensch. Proc. Ser, $A 84$ (1981), 39-66.

3. L. Danzer, Three-dimensional analogs of the planar Penrose tilings and quasicrystals, Discrete Math. 78 (1989), 1-7.

4. Y. Fukamo, T. Ishimasa, and H.-U. Nissen, New ordered state between crystalline and amorphous in Ni-Cr particles, Phys. Rev. Lett. 55 (1985), 511-513.

5. B. Grünbaum and G. C. Shephard, Tilings and Patterns, Freeman, New York, 1987.

6. P. Kramer and R. Neri, On periodic and non-periodic space fillings on $\mathbb{E}^{m}$ obtained by projections, Acta Cryst. A 40 (1984), 580-587. 
7. R. D. Nelson, Quasicrystals, Sci. Amer. 255 (1986), 32-41.

8. R. Penrose, Pentaplexity, Eureka 39 (1978), 16-22.

9. M. Senechal and J. Taylor, Quasicrystals: the view from Les Houches, Math. Intelligencer 12 (1990), 54-64.

10. D. Shechtman, I. Bleck, D. Gratias, and J. W. Cahn, Metallic phases with long-range orientational order and no translational symmetry, Phys. Rev. Lett. 53 (1988), 105-112.

Received March 5, 1990, and in revised form September 10, 1990. 